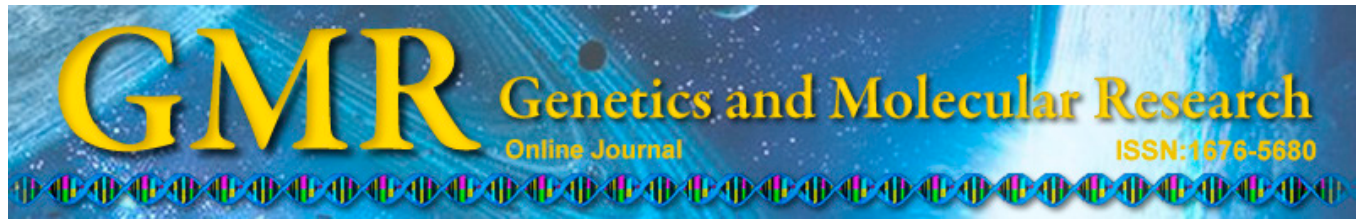

\title{
Elevated serum homocysteine level in the development of diabetic peripheral neuropathy
}

\author{
L.Q. Zheng ${ }^{1}$, H.L. Zhang ${ }^{2}$, Z.H. Guan ${ }^{1}$, M.Y. Hu' ${ }^{1}$, T. Zhang ${ }^{1}$ and S.J. Ge ${ }^{2}$ \\ ${ }^{1}$ Department of Endocrinology and Metabolism, \\ Wenzhou Hospital of Traditional Chinese Medicine, Wenzhou, China \\ ${ }^{2}$ Department of Endocrinology and Metabolism, \\ The First Affiliated Hospital of Wenzhou Medical University, Wenzhou, China \\ Corresponding author: S.J. Ge \\ E-mail: geshengjie107@126.com
}

Genet. Mol. Res. 14 (4): 15365-15375 (2015)

Received June 26, 2015

Accepted September 27, 2015

Published November 30, 2015

DOI http://dx.doi.org/10.4238/2015.November.30.14

\begin{abstract}
The development of diabetic peripheral neuropathy (DPN) is always followed by changes in vascular endothelial cells that are related to the reactivity of the homocysteine (Hcy) sulfhydryl group. In this meta-analysis, we investigated the association of Hcy with the pathogenesis and progression of DPN. We screened the Embase, Ovid, PubMed, Web of Science, Wangfang, and China National Knowledge Infrastructure databases. All analyses were performed by using the STATA software, version 12.0 (StataCorp, College Station, TX, USA) and the Comprehensive Meta-analysis 2.0 software (Biostatic Inc., Englewood, NJ, USA). The standardized mean difference (SMD) and $95 \%$ confidence interval $(95 \% \mathrm{CI})$ were further calculated. The electronic literature search identified six articles that included 603 patients with DPN and 687 healthy controls. The pooled SMD of those six studies revealed that increased serum levels of Hcy may be correlated with DPN $(\mathrm{SMD}=1.23,95 \% \mathrm{CI}$ : 1.09-1.36, $\mathrm{P}<0.001)$. Subgroup analysis
\end{abstract}


according to ethnicity indicated that high serum Hcy levels might be an important risk factor for DPN in both Asian and Caucasian populations (Asians: SMD $=0.62,95 \% \mathrm{CI}$ : 0.45-0.79, $\mathrm{P}<0.001$; Caucasians: SMD $=2.32,95 \% \mathrm{CI}: 2.10-2.55, \mathrm{P}<0.001$; respectively). Elevated serum levels of Hcy indicate the risk of development of DPN in patients, suggesting that Hcy levels could be used as a marker for new therapeutic approaches to DPN.

Key words: Homocysteine; Diabetic peripheral neuropathy; Vascular endothelial cell; Sulfhydryl amino acid; Pathogenesis; Meta-analysis

\section{INTRODUCTION}

Diabetic peripheral neuropathy (DPN) is a disease whose symptoms are related to dysfunction of peripheral nerves, such as demyelination, axonal atrophy, blunted regenerative potential, and loss of peripheral nerve fibers, that occur specifically in patients with diabetes (Farmer et al., 2012). The number of diabetic patients worldwide is expected to increase from 171 million in 2000 to at least 366 million by 2030, on the basis of the World Health Organization's statistics (Erbas et al., 2011). Furthermore, considering the specific morbidity and mortality of DPN in diabetic patients, in the United Kingdom, the estimated prevalence of painful DPN was $26.4 \%$ among diabetic patients who, in turn, comprise $4.5 \%$ of the entire population (Erbas et al., 2011). In Korea, the prevalence of DPN has been documented to have increased from 14.1 to $54.5 \%$ in the last five decades, based on the study population and diagnostic criteria (Won et al., 2014). It was estimated that DPN was diagnosed in three million people in the United States in 2010 (Wolff et al., 2010). The burden of DPN is considerable for both patients and the society; that is, despite polypharmacy and high use of resources, the discomfort of patients persists and may be a risk factor for foot ulceration, which finally causes lower-extremity amputation and can lead to limitations in daily activities (Tesfaye et al., 2011; Kim et al., 2014). It was estimated that the excess health-care costs in painful DPN ranged from $<\$ 1000$ to $>\$ 8000$ due to the different personal conditions of patients (Dworkin et al., 2011). The pathogenesis of DPN is widely accepted to be poorly understood and seems to be multifactorial with suggested etiologies such as microvascular abnormalities, nitric oxide (NO) deficiency, acute or chronic hyperglycemia, nerve compression, and environmental and genetic factors (Pieber et al., 2010). Until recently, many experts related the pathogenesis of DPN to metabolic processes (Tesfaye and Selvarajah, 2012). DPN was reported to result from various biochemical perturbations, including oxidative stress, polyol pathway activation, increase in advanced glycation end products and the activation of their receptors, hypoxia, ischemia, and elevated cytokines (Chan et al., 2011; Singh et al., 2014). Several studies have reported the relation between DPN and plasma homocysteine (Hcy) because of the underlying changes in vascular endothelial cells due to the reactivity of the sulfhydryl group of Hcy (Ambrosch et al., 2001; Jianbo et al., 2011; El Boghdady and Badr, 2012; Gonzalez et al., 2012).

Hcy, which is secreted through the metabolism of methionine, is a sulfhydryl amino acid dependent on folic acid, pyridoxine, and vitamin $\mathrm{B}_{12}$; because of this strong association, Hcy is often considered a sensitive marker of these vitamins (Tassino et al., 2009). Furthermore, 
serum Hcy is susceptible to a wide range of other factors such as diet, drug use, lifestyle, and certain genetic abnormalities (Jianbo et al., 2011). In clinical practice, Hcy has been suggested to be a predictive factor of cognitive dysfunctions, dementia, neurodegenerative pathologies, and neurovascular alterations such as in Parkinson's and Alzheimer's disease (Tassino et al., 2009). In addition, some studies have also demonstrated that the reactivity of the plasma Hcy levels may be implicated in a series of diabetic complications such as foot ulceration, cerebrovascular disease, coronary artery disease, stroke, thrombosis, and atherosclerosis (Tassino et al., 2009; Vinukonda et al., 2009; Gonzalez et al., 2010; Gazzaruso et al., 2012). Moreover, recent studies have revealed that there is a significant association between plasma Hcy concentrations and the occurrence and grade of DPN (Gonzalez et al., 2012). Elevated plasma Hcy values, which could subsequently potentially affect the mechanisms of vascular endothelial cells, could contribute to the presence of DPN because of the resultant circulation impairment, nerve damage, and nutrient deficit; the risk of DPN in patients with high Hcy is twice greater than in those with low Hcy values (Wile and Toth, 2010; Gonzalez et al., 2012). Furthermore, several studies have suggested that increasing serum levels of Hcy worsen the degree of DPN: the higher the plasma Hcy concentration, the higher the grade of DPN (Jianbo et al., 2011). Therefore, we could hypothesize that serum Hcy levels may play a pivotal role in the prediction and even diagnosis of DPN (Wile and Toth, 2010). Multiple previous studies have shown that elevated serum levels of Hcy have a close association with DPN, and Hcy could be used as a sensitive and effective predictor of DPN; however, other studies have presented inconsistent results (Wile and Toth, 2010; Gonzalez et al., 2012). Therefore, we conducted this metaanalysis to further evaluate the role of plasma Hcy concentration as a biomarker for predicting DPN, while investigating its potential role in the pathogenesis, diagnosis, and treatment of the disease to improve its management and prevent further complications.

\section{MATERIAL AND METHODS}

\section{Search strategy}

Related studies were searched from Embase, Ovid, PubMed, Web of Science, Wangfang, and China National Knowledge Infrastructure databases. All published articles assessing the correlations between Hcy serum levels and DPN from the oldest publication data up to April 30, 2014 were extracted by using the search terms "Homocysteine" or "Homocysteine" or "2-amino-4-mercaptobutyric acid" or "Hcy" or "Homocysteine," and "Diabetic Neuropathies" or "Diabetic Neuropathies" or "Diabetic peripheral neuropathy" or "DPN" or "painful diabetic peripheral neuropathy" or "PDPN." The search was not language restricted. Manual search was also done to find additional potentially relevant articles.

\section{Selection criteria}

All case-control studies about the association of Hcy serum levels with DPN as a main outcome were primarily considered. In the trials, the presence and severity of DPN in patients were confirmed according to the standard Neuropathy Symptom Score and Neuropathy Disability Score criteria (Inanir et al., 2013). Moreover, factors associated with Hcy serum levels were also included in the initial review of articles. Studies that have no data about the number 
of DPN cases or have insufficient information about the serum Hcy levels were excluded. Extracted trials with a minimum number of included cases of $>15$ were included in the analysis. However, extracted studies that were duplicates or had incomplete data were excluded. If multiple studies involved the same population, only the most recent or complete study was included after a careful reexamination.

\section{Data extraction}

Two investigators extracted information by using a predetermined form supplemented with inclusion and exclusion criteria independently, and the following data were abstracted from each study: first author, publication time, age and gender, ethnicity and country, source of publication, study type, source of controls, sample size, study design, detection method of Hcy serum levels, and Hcy expression levels. Any disagreements in our meta-analysis were solved by consensus. All authors approved the final enrollment of the studies.

\section{Statistical analysis}

For the purpose of supplying quantitative evidence of all included trials and to minimize the variance of the summary, we carried out this meta-analysis by applying a fixedeffect or a random-effect model. The random-effect model was used when heterogeneity exists among studies, whereas the fixed-effect model was used when there was no statistical heterogeneity. The summary standardized mean difference (SMD) and $95 \%$ confidence interval $(95 \% \mathrm{CI})$ were calculated for the case $v s$ control category of Hcy serum levels by using the Ztest. Heterogeneity was evaluated by using Cochran's $Q$-statistic (Jackson et al., 2012). When the statistical power of Cochran's $Q$-statistic was low, the $I^{2}$ test was also applied to assess the heterogeneity across studies (Peters et al., 2006). A subgroup meta-analysis based on ethnicity, sample size, and detection method was applied to investigate the potential sources of heterogeneity among the enrolled trials. To measure the impact of a single study on the overall results, a one-way sensitivity analysis was done to ensure that no one study was completely responsible for the overall results. Furthermore, funnel plot, classic fail-safe N, and the Egger tests were applied to detect any publication bias, to further confirm the original result (Egger et al., 1997; Wikstrom et al., 2009). All tests were two-sided, and P values $<0.05$ were considered to be statistically significant. Two investigators separately performed the statistical analysis by using the STATA 12.0 software (StataCorp, College Station, TX, USA) and the Comprehensive Meta-analysis 2.0 software (Biostatic Inc., Englewood, NJ, USA), and reached an agreement eventually.

\section{RESULTS}

\section{Characteristics of studies included}

The electronic literature search supplemented with a manual search identified a sum of 106 relevant articles. After excluding 4 duplicates; 9 letters, reviews, or meta-analysis; 15 nonhuman studies; and 21 studies not related to the research topics, 57 full-text articles remained. After further selecting and assessing the remaining articles, 7 studies were excluded 
for not being a case-control study, 16 for being irrelevant to Hcy, 25 for being irrelevant to DPN, and 3 studies were removed for having incomplete or weakly correlated data. A total of six papers that included 603 patients with DPN and 687 healthy controls were finally selected on the basis of our inclusion criteria (Ambrosch et al., 2001; Cohen et al., 2001; Abdella et al., 2002; Li et al., 2011a; El Boghdady and Badr, 2012; Gonzalez et al., 2012). The publication years were between 2001 and 2012. All trials had a case-control study design relating to the association between Hcy serum levels and DPN in Asian (three trials) and Caucasian (three trials) populations. The detection methods included immunoassay (Advia Centaur HCY2 assay, enzyme-linked immunosorbent assay, fluorescence polarization immunoassay, and chemiluminescent magnetic immunoassay) and chromatography (gas chromatography/mass spectrometry and high-performance liquid chromatography). None of the studies deviated from the Hardy-Weinberg equilibrium (all $\mathrm{P}>0.05$ ). Table 1 shows the baseline characteristics and quality of all the selected trials.

\section{Serum levels of Hcy in DPN}

Six studies referred to the Hcy serum levels in DPN. The main findings of the correlation of the levels of Hcy and DPN are presented in Figure 1. The random-effects model was used to test the heterogeneity $(\mathrm{P}<0.001)$. The meta-analysis results suggested a positive association between serum levels of Hcy and DPN (SMD = 1.23, 95\%CI: 1.09-1.36, P < 0.001). The subgroup analysis according to ethnicity showed that high serum levels of Hcy might be the main risk factor for DPN in both Asians and Caucasians (all $\mathrm{P}<0.05$ ) (Figure 2). A further subgroup analysis based on sample size showed obviously positive associations between the levels of Hcy and DPN in both small and large subgroups (all $\mathrm{P}<0.05$ ). In addition, Figure 2 shows that the Hcy serum levels were significantly related to DPN on the basis of immunoassay and chromatography methods (all $\mathrm{P}<0.05$ ).

\section{Sensitivity analysis and publication bias}

Sensitivity analysis was carried out to assess the stability of this meta-analysis when selected studies were individually deleted to reflect the significance of the overall effect size (SMDs). As shown in Figure 3, no single publication was completely responsible for the overall results. The symmetrical funnel plot in Figure 4 shows that no publication bias existed in our meta-analysis, which was further confirmed by the classic fail-safe $\mathrm{N}$ and Egger's tests.

\section{DISCUSSION}

Our aim was to investigate the correlation of serum Hcy levels with the prevalence and grade of DPN. In this meta-analysis, we observed that plasma total Hcy levels significantly increased in DPN patients, suggesting that plasma Hcy level can be considered a risk factor for the development and grade of DPN. However, the exact mechanism by which plasma Hcy level leads to the development and progression of DPN is still largely unknown. As one of the most common chronic complications of diabetes, the pathogenesis of DPN is closely related to metabolic disturbance, vascular endothelial dysfunction, neurotrophic disturbances, oxidative stress, and autoimmune factors (Tesfaye and Selvarajah, 2012). Hcy is well known to be 
L.Q. Zheng et al.

15370

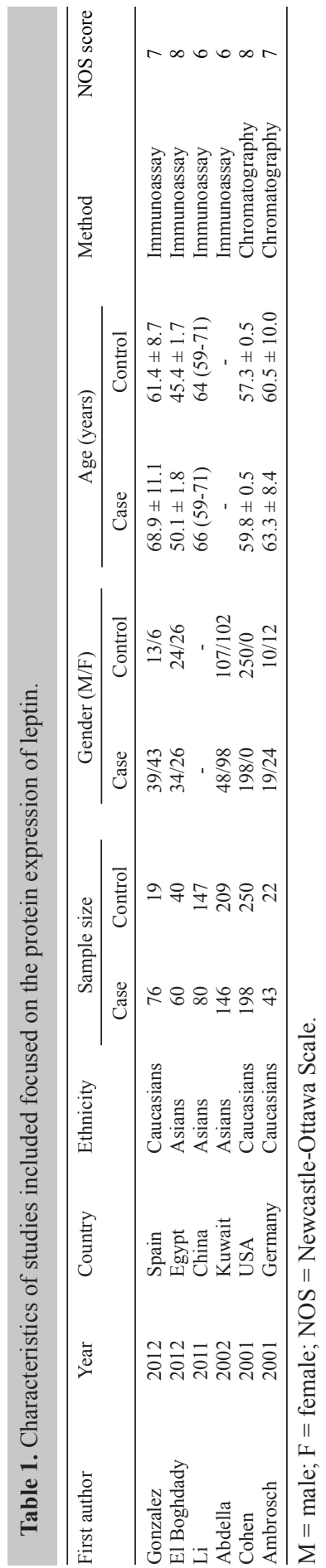

Genetics and Molecular Research 14 (4): 15365-15375 (2015) 


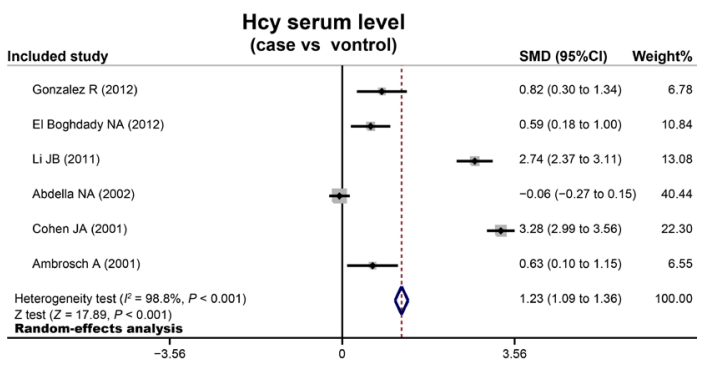

Figure 1. Forest plots for the differences of serum homocysteine (Hcy) level between diabetic peripheral neuropathy patients and control subjects.
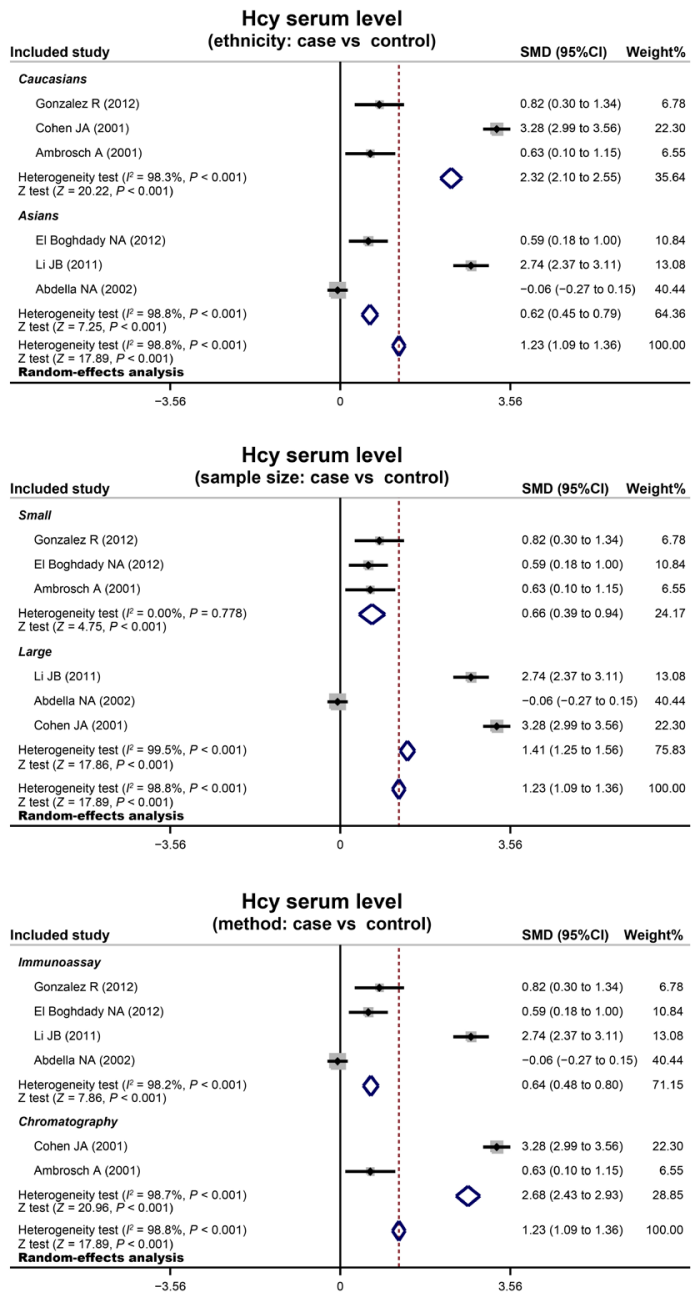

Figure 2. Subgroup analyses for the differences of serum homocysteine (Hcy) level between diabetic peripheral neuropathy patients and the healthy controls. 


\section{Hcy serum level}

(case vs control)

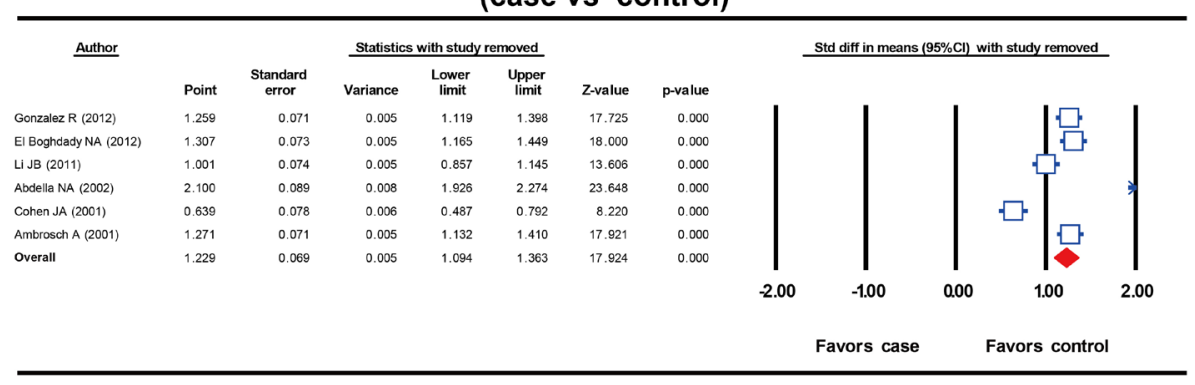

Figure 3. Sensitivity analysis of the pooled odds ratio coefficients for the differences of serum homocysteine (Hcy) level between diabetic peripheral neuropathy patients and the healthy controls.

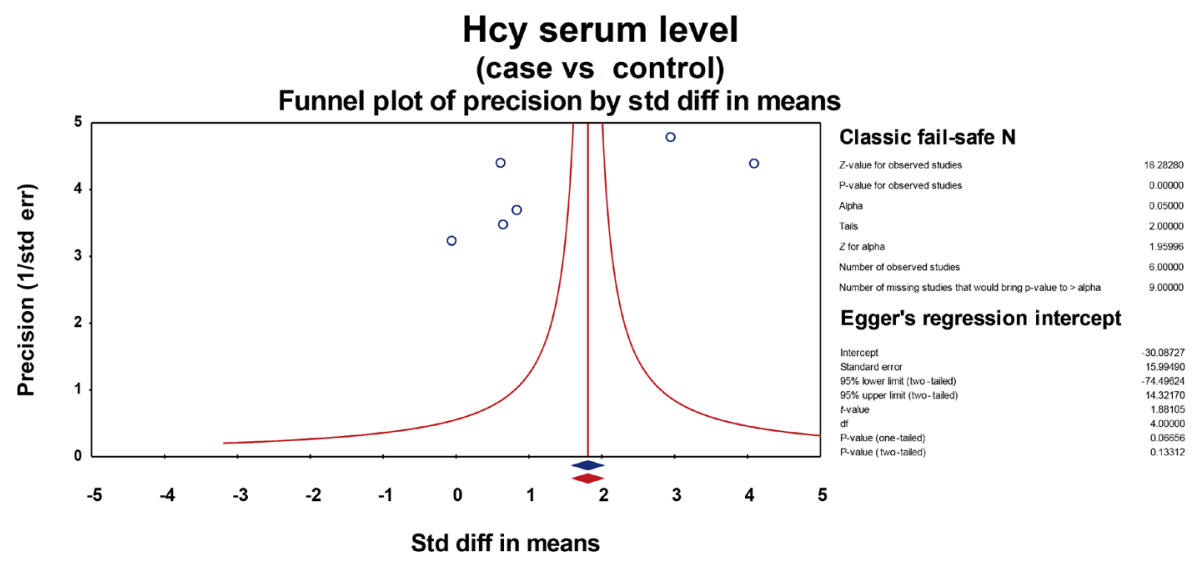

Figure 4. Funnel plot of publication biases for the differences of serum homocysteine (Hcy) level between diabetic peripheral neuropathy patients and the healthy controls.

a nonprotein amino acid containing sulfur and can function as an intermediate in the metabolism of methionine (Huang et al., 2013). Meanwhile, abnormally high serum levels of Hcy was reported to be a significant risk factor for the progression of multiple diseases, including fractures, thrombosis, and neuropsychiatric illness (Leboff et al., 2009; Dionisio et al., 2010). More specifically, increased plasma Hcy levels have been recognized to be correlated with many complications, including endothelial dysfunction, inflammation, insulin resistance, prothrombotic state, macroangiopathy, and nephropathy, in patients with diabetes (Ndrepepa et al., 2008). Furthermore, elevated Hcy serum concentrations can induce oxidative stress, which has a cytotoxic effect; causes oxidative damage in endothelial cells; and can inhibit the production of NO, which results in arteriosclerosis and thrombosis (Tyagi et al., 2005). Elevated Hcy serum concentration and decreased NO level can cause functional damage to vascular smooth muscle and reduce the blood flow in the endothelium, which is considered to be related to neurotrophic disturbances, nerve fiber pathology, and nerve fiber loss (El Boghdady and 
Badr, 2012). In addition, it has been indicated that high serum Hcy levels increase the risk of endothelial injury, disorders of glucose and lipid metabolism, and neural lesions, which might play an important role in the development and progression of DPN (Gonzalez et al., 2012). In this regard, increased Hcy serum levels can lead to the occurrence and development of DPN in patients with diabetes. Li et al. (2011b) demonstrated that plasma Hcy levels were significantly higher in diabetic neuropathy patients than in those without diabetic neuropathy, implying that increased plasma Hcy level was an independent factor for the increase in the risk of DPN. We also carried out a subgroup analysis based on ethnicity to evaluate the correlation between the serum Hcy level and the development of DPN. The results of the stratified analysis showed that increased serum level of Hcy has a significant correlation with the development of DPN in both Caucasians and Asians. Altogether, our findings were consistent with previous studies demonstrating that the serum Hcy level was increased in the diabetes patients with DPN, showing that serum levels of Hcy may be strongly linked with the development and progression of DPN and can be used as a potential biomarker for DPN development.

In this meta-analysis, the inclusion of unpublished studies on Hcy and DPN may strengthen the credibility of our results. Moreover, another advantage may be that all studies included have an acceptable quality (a score of at least 7). In addition, no publication bias was discovered when applying the Egger test, suggesting that the statistical data extracted from those enrolled trials may approximate the actual results. However, it should be noted that there were several limitations in the present analysis. First, as this analysis was based on literature reports with predominantly positive results, our results about the relation between Hcy and DPN might be overestimated. The second limitation may be the presence of unignorable biases. Nevertheless, as mentioned above, no publication bias was detected in Egger's test, and we did not consider unpublished articles and abstracts entirely because the required data for inclusion were unavailable. Third, although comprehensive data were extracted for statistical analysis, the studies selected for this meta-analysis included multiple ethnic populations, and the country, age, gender, and lifestyle were all variable. All of the above information we used could have resulted in an inconsistent outcome. Fourth, only studies written in Chinese or English were enrolled in the meta-analysis although we did not apply a language restriction in the inclusion criteria.

In conclusion, our results showed that elevated serum Hcy levels may be closely correlated with the risk of DPN development and progression, in both Asians and Caucasians. The data suggested that the development of strategies against Hcy could lead to a reasonable therapeutic treatment program for DPN. To confirm our findings, more studies on non-Asian and non-Caucasian populations with a more appropriate multivariate analysis are necessary.

\section{Conflicts of interest}

The authors declare no conflict of interest.

\section{ACKNOWLEDGMENTS}

We thank all the reviewers for their valuable suggestions that helped improved this article. 


\section{REFERENCES}

Abdella NA, Mojiminiyi OA, Akanji AO and Moussa MA (2002). Associations of plasma homocysteine concentration in subjects with type 2 diabetes mellitus. Acta Diabetol. 39: 183-190.

Ambrosch A, Dierkes J, Lobmann R, Kuhne W, et al. (2001). Relation between homocysteinaemia and diabetic neuropathy in patients with Type 2 diabetes mellitus. Diabet. Med. 18: 185-192.

Chan L, Terashima T, Urabe H, Lin F, et al. (2011). Pathogenesis of diabetic neuropathy: bad to the bone. Ann. N. Y. Acad. Sci. 1240: 70-76.

Cohen JA, Jeffers BW, Stabler S, Schrier RW, et al. (2001). Increasing homocysteine levels and diabetic autonomic neuropathy. Auton. Neurosci. 87: 268-273.

Dionisio N, Jardin I, Salido GM and Rosado JA (2010). Homocysteine, intracellular signaling and thrombotic disorders. Curr. Med. Chem. 17: 3109-3119.

Dworkin RH, Panarites CJ, Armstrong EP, Malone DC, et al. (2011). Healthcare utilization in people with postherpetic neuralgia and painful diabetic peripheral neuropathy. J. Am. Geriatr. Soc. 59: 827-836.

Egger M, Davey Smith G, Schneider M and Minder C (1997). Bias in meta-analysis detected by a simple, graphical test. BMJ 315: 629-634.

El Boghdady NA and Badr GA (2012). Evaluation of oxidative stress markers and vascular risk factors in patients with diabetic peripheral neuropathy. Cell Biochem. Funct. 30: 328-334.

Erbas T, Ertas M, Yucel A, Keskinaslan A, et al. (2011). Prevalence of peripheral neuropathy and painful peripheral neuropathy in Turkish diabetic patients. J. Clin. Neurophysiol. 28: 51-55.

Farmer KL, Li C and Dobrowsky RT (2012). Diabetic peripheral neuropathy: should a chaperone accompany our therapeutic approach? Pharmacol. Rev. 64: 880-900.

Gazzaruso C, Coppola A, Montalcini T, Baffero E, et al. (2012). Lipoprotein(a) and homocysteine as genetic risk factors for vascular and neuropathic diabetic foot in type 2 diabetes mellitus. Endocrine 41: 89-95.

Gonzalez R, Pedro T, Real JT, Martinez-Hervas S, et al. (2010). Plasma homocysteine levels are associated with ulceration of the foot in patients with type 2 diabetes mellitus. Diabetes Metab. Res. Rev. 26: 115-120.

Gonzalez R, Pedro T, Martinez-Hervas S, Civera M, et al. (2012). Plasma homocysteine levels are independently associated with the severity of peripheral polyneuropathy in type 2 diabetic subjects. J. Peripher. Nerv. Syst. 17: 191-196.

Huang T, Ren J, Huang J and Li D (2013). Association of homocysteine with type 2 diabetes: a meta-analysis implementing Mendelian randomization approach. BMC Genomics 14: 867.

Inanir A, Basol N, Karakus N and Yigit S (2013). The importance of association between angiotensin-converting enzyme (ACE) Gene I/D polymorphism and diabetic peripheral neuropathy. Gene 530: 253-256.

Jackson D, White IR and Riley RD (2012). Quantifying the impact of between-study heterogeneity in multivariate metaanalyses. Stat. Med. 31: 3805-3820.

Jianbo L, Yuche C, Ming S, Jingrong T, et al. (2011). Association of homocysteine with peripheral neuropathy in Chinese patients with type 2 diabetes. Diabetes Res. Clin. Pract. 93: 38-42.

Kim SS, Won JC, Kwon HS, Kim CH, et al. (2014). Prevalence and clinical implications of painful diabetic peripheral neuropathy in type 2 diabetes: results from a nationwide hospital-based study of diabetic neuropathy in Korea. Diabetes Res. Clin. Pract. 103: 522-529.

Leboff MS, Narweker R, LaCroix A, Wu L, et al. (2009). Homocysteine levels and risk of hip fracture in postmenopausal women. J. Clin. Endocrinol. Metab. 94: 1207-1213.

Li JB, Cheng YC, Shi M, Tang JR, et al. (2011a). Association of homocysteine with peripheral neuropathy in Chinese patients with type 2 diabetes. Diabetes Res. Clin. Pract. 93: 38-42.

Li JB, Cheng YZ, Shi M, Zhang HM, et al. (2011b). The relationship between plasma homocysteine levels and diabetic peripheral neuropathy. Chin. J. Intern. Med. 50: 14-17.

Ndrepepa G, Kastrati A, Braun S, Koch W, et al. (2008). Circulating homocysteine levels in patients with type 2 diabetes mellitus. Nutr. Metab. Cardiovasc. Dis. 18: 66-73.

Peters JL, Sutton AJ, Jones DR, Abrams KR, et al. (2006). Comparison of two methods to detect publication bias in metaanalysis. JAMA 295: 676-680.

Pieber K, Herceg M and Paternostro-Sluga T (2010). Electrotherapy for the treatment of painful diabetic peripheral neuropathy: a review. J. Rehabil. Med. 42: 289-295.

Singh R, Kishore L and Kaur N (2014). Diabetic peripheral neuropathy: current perspective and future directions. Pharmacol. Res. 80: 21-35.

Tassino M, Campos TF and Guerra RO (2009). Homocysteine (Hcy) and cognitive performance in a population sample of elderly Brazilians. Arch. Gerontol. Geriatr. 48: 142-145. 
Tesfaye S and Selvarajah D (2012). Advances in the epidemiology, pathogenesis and management of diabetic peripheral neuropathy. Diabetes Metabol. Res. Rev. 28 (Suppl 1): 8-14.

Tesfaye S, Vileikyte L, Rayman G, Sindrup S, et al. (2011). Painful diabetic peripheral neuropathy: consensus recommendations on diagnosis, assessment and management. Diabetes Metabol. Res. Rev. 27: 629-638.

Tyagi N, Sedoris KC, Steed M, Ovechkin AV, et al. (2005). Mechanisms of homocysteine-induced oxidative stress. Am. J. Physiol. Heart Circ. Physiol. 289: H2649-H2656.

Vinukonda G, Shaik Mohammad N, Md Nurul Jain J, Prasad Chintakindi K, et al. (2009). Genetic and environmental influences on total plasma homocysteine and coronary artery disease (CAD) risk among South Indians. Clin. Chim. Acta 405: 127-131.

Wikstrom EA, Naik S, Lodha N and Cauraugh JH (2009). Balance capabilities after lateral ankle trauma and intervention: a meta-analysis. Med. Sci. Sports Exerc. 41: 1287-1295.

Wile DJ and Toth C (2010). Association of metformin, elevated homocysteine, and methylmalonic acid levels and clinically worsened diabetic peripheral neuropathy. Diabetes Care 33: 156-161.

Wolff RF, Bala MM, Westwood M, Kessels AG, et al. (2010). 5\% lidocaine medicated plaster in painful diabetic peripheral neuropathy (DPN): a systematic review. Swiss Med. Wkly. 140: 297-306.

Won JC, Kim SS, Ko KS and Cha BY (2014). Current status of diabetic peripheral neuropathy in Korea: report of a hospital-based study of type 2 diabetic patients in Korea by the Diabetic Neuropathy Study Group of the Korean Diabetes Association. Diabetes Metabol. J. 38: 25-31. 